\title{
The Role of Culture in Effective Intervention Design, Implementation, and Research: Its Universal Importance
}

\author{
Spero M. Manson ${ }^{1}$
}

Published online: 28 October 2019

(C) Society for Prevention Research 2019

\begin{abstract}
This special issue on Promoting Health Equity through Rigorous, Culturally Informed Intervention Science: Innovations with Indigenous Populations in the United States is a welcome addition to the growing recognition that culture is fundamental to effective intervention design, implementation, and research, in this instance among Indigenous peoples of North America and the Pacific. The articles herein summarize some of the priorities, findings, and lessons learned through work conducted by researchers in the National Institutes of Health Intervention Research to Improve Native American Health (IRINAH) consortium. The lessons learned encourage rethinking of the epistemological imperatives of our science; recognizing the rich, important variation in the lived experience of Indigenous peoples; and a greater appreciation for the critical role this experience plays in conceptualizing, designing, and operationalizing effective interventions. In this regard, the authors return us to a nearly 3 decades' old, but largely unrealized commitment by the National Institutes of Health to the thoughtful inclusion of underrepresented racial and ethnic minorities in its sponsored research as a means of improving the clinical and implementation sciences. The findings summarized in this special issue demonstrate the value of such efforts and fit well within existing paradigms that emphasize the importance of culture for maximizing the relevance and impact of this work. Yet the remaining challenge is to promote this agenda in ways that invite others to contribute in equally deliberate and informed ways, and thereby advance the field at large.
\end{abstract}

This special issue is a welcome addition to the growing recognition that culture is fundamental to effective intervention design, implementation, and research, in this instance among Indigenous peoples of North America and the Pacific. The irony, from my perspective, is that the lessons learned and reported herein are not limited to this special population, and have import for all humankind, regardless of gender, age, language, religion, or race/ethnicity. Culture is intrinsic to our very nature: to all of us. It shapes our perception of the world; it is the vehicle by which we make and share meaning; it binds us to one another. The content and emphases of our cultural lives may vary, but the fact we are cultural beings does not. Accordingly, my comments address this second of the four themes that cross-cut every contribution to this special issue, with particular relevance to culture as the center piece.

Spero M. Manson

spero.manson@cuanschutz.edu

1 Centers for American Indian and Alaska Native Health, Colorado School of Public Health, University of Colorado, Anschutz Medical Campus, 13055 E. 17th Avenue, Mail Stop F800, Aurora, CO 80045, USA
Toward this end, let me share several stories that embody the above points.

Forty years ago, early in my career at the Oregon Health Sciences University, I taught a course entitled "Health and Healing in the Context of Culture" to medical students and residents. As a young anthropologist, I labored at the intersection of medicine, health, and culture. I sought to understand and, in turn, convey to physicians-in-training the rich array of views on illness and its treatment as well as wellness and its maintenance that abound among different peoples of the world. The course introduced representatives of these views, ranging from those most likely to be familiar to the studentstheir allopathic, homeopathic, and naturopathic counterparts - to those who typically fell beyond their experience - acupuncturists, curanderos, Buddhist monks, and traditional American Indian healers. Under my direction, students interviewed these practitioners, with an eye toward discovering the latter's explanatory models of health and illness as a first step toward comparative analyses of different systems of healing. The session with a Kul Wicasa Oyate (Lower Brule Sioux) medicine man offered one lesson directly relevant to the theme of culture as a central organizing principle in IRINAH research and intervention implementation. 
The healer in question was Devere Eastman, the grandson of Charles Eastman, MD, among the first American Indian physicians, and who tended to survivors of the infamous Wounded Knee Massacre of December 1890 on the Pine Ridge Indian Reservation. Part way through the class period, Mr. Eastman realized the students failed to comprehend his description, in words, of what it was that he did, how his practice worked, or evidence for its effectiveness. Frustrated, he stopped, grasped the right-hand braid of his long hair and announced:

“Here, look at my braid. In our people's way of looking at the world, it represents the human condition. This braid is woven from three strands."

As he began to unravel one strand from the other two, he continued:

"This strand stands for the body. In the Western world, you give this to your surgeons and cardiologists, to "real doctors", to care for."

He let it drop, selected the second strand, and separated it from the third. Holding the former, Mr. Eastman observed:

"This strand stands for the mind. You give it to your psychiatrists and psychologists to care for."

Letting go, it fell, and he seized the third strand. Holding it aloft, he declared:

"This strand represents our spirit. You give it to your priests and ministers to tend."

Then, allowing all three strands to dangle, each apart from one another, he asked:

"But where, then, lies the human condition?"

Immediately reaching to his left-hand braid, he continued:

"In my world, among us, the 'two-legged', mind, body, and spirit are woven together. They are inseparable. Once they become undone, we lose who we are. Among the Lakota, I help weave these aspects of ourselves together. The tools I have, the skills I possess, and my heart serve this mission."

Metaphor is a powerful vehicle for conveying the essence of the world around us. In this instance, we are reminded of the limits of Cartesian dualism, of the separation of self into distinct components that more readily lend themselves to analysis. But we then struggle to reassemble them in meaningful ways that can inform, in this instance, preventive interventions (Gold 1985; Thibaut 2018). Several contributions to this special issue speak directly to such challenges and illustrate how we might proceed, both in principle as well as practice (Dickerson et al. 2018; Ivanich et al. 2018; Rasmus et al. 2018; Walters et al. 2018).

Years later, another important lesson underscoring the fundamental importance of culture for implementation science surfaced in my work among the Hopi which sought to understand the nature and experience of depression cross-culturally. I interviewed an older Hopi woman as she wove a basket from a wide range of brightly dyed grass fibers. The bottom of the basket featured a dark blue circle, surrounded in turn by four concentric circles, each a different color from the others. I asked her about the meaning of the design; smiling, this elder allowed that she was "... recreating myself...". That being the case, "... which circles", she asked, "did I believe represented her, her family, community, the broader world, and the Creator?" I deferred to her, claiming not to know. Gently chiding me, she revealed that the dark blue circle at the center represented the Creator, followed by the fauna and flora of the world, then community, family, and the largest circle at the outer margins symbolized herself. "I am all these things. I am their sum."

I subsequently showed a photograph of her basket to medical students and residents in yet another course I taught at the Oregon Health Sciences University. I put to them the very same question this Hopi woman had asked me. Scoffing at such a seemingly simple quiz, one student replied; "It's obvious. The dark blue circle at the center represents the woman who is weaving the basket, followed by her family, community, the broader world, and the Creator at the outer edge". Asking his fellow students to raise their hands if they agreed with him, all did, a perfect demonstration of reliability in perception, but error with respect to validity. Just because we may agree in our interpretation of the thoughts, feelings, and behaviors deemed central to prevention does not mean that these interpretations necessarily align with those from other populations.

This example illustrates one of the major cultural tensions underpinning approaches to implementation science: namely, the primacy of egocentric versus sociocentric definitions of self and identity as one moves across different cultural contexts. Dickerson et al. (2018), particularly in their discussion entitled Honoring the Medicine, describe the friction that arises - at the points of conceptualization, adaptation, engagement, delivery, evaluation, and even review for funding — as a consequence of such tensions. Returning to the Hopi basketmaker, one can imagine the inadequacy of interventions that attend primarily to her thoughts, feelings, and behaviors absent consideration of the broader social and psychological landscape within which they are situated. Again, Western science has begun to recognize such constraints on its long-held assumptions (Kirmayer 2007). Witness the growing interest in 
multi-level study designs and modeling to address the increasingly complex context of this lived experience (Jernigan et al. 2018). But it has a long way to go.

A third and final example touches upon the loci of thought, emotion, and being. The aforementioned study of depression also took place among the Sahaptin people of northeastern Oregon. Careful inquiry revealed that their construction of self was firmly rooted in one's heart. Thought and emotion were expressed as conditions of the heart: as a "black heart", "sad heart", "fearful heart", "joyous heart", "lonely heart", "confused heart", and "deliberate heart" (Manson 1994). Our subsequent translation of a cognitive-behavioral approach to preventing depression among physically impaired older tribal members had to rework the attendant procedures - e.g., covert rehearsal, reframing threat as opportunity, progressive relaxation (Manson and Brenneman 1995). These very different assumptions about the resident self, about meaningful states, and how to access, much less alter them, required a dramatic revision of intervention mechanics and content.

This challenge was also thoughtfully anticipated by several articles in the special issue, which highlighted the process of translation as much more than simply discovering and recasting intervention content and format in terms of cultural analogs (Dickerson et al. 2018; Ivanich et al. 2018). Two examples, Bii-Zin-Da-De-Dah ("Listening to One Another") and Thiwáhe Gluwášsakapi ("Sacred Home Where Families are Made Strong"), illustrate how, among the Ojibwe and Lakota, respectively, the authors combined powerful notions of self, located in the broader inter-subjective lives of these tribal communities, with the intersection of family and socialization processes emphasized by Strengthening Families Program (Spoth and Redmond 2002) to intervene in ways more potent than either alone would likely have engendered.

Before concluding, let me locate these observations within the broader context of National Institute of Health (NIH) sponsorship of the discovery, acquisition, dissemination, and application of knowledge critical to prevention and implementation science. It is especially appropriate given that the NIH initiative known as Health Intervention Research to Improve Native American Health (IRINAH) gave rise to the articles contained in this special issue.

In January 10, 1992, the NIH published, within its Guide for Grants and Contracts, special instructions to applicants regarding implementation of new policies concerning the inclusion of women and minorities in clinical research study populations. This, then, new policy spoke to the underrepresentation of racial and ethnic minorities in the science of the times, and strongly argued for closer attention to their inclusion in NIH-sponsored research as a means of improving said science. The circumstances leading to this policy change, as well as the ensuing response on the part of the National Institutes of Health and Department of Health and Human Services to its implementation, are well known.
Unfortunately, despite such a remarkable advance, the vigilance necessary to realize the promise embodied in this change waned. Indeed, as a long-standing member of several established NIH study sections charged with determining the scientific merit of thousands of grant applications submitted for prospective funding, I witnessed firsthand the gradual attenuation of this policy of inclusion. Study sections struggled with its operationalization; we lacked carefully crafted examples of how adherence to this policy could be accomplished in a pragmatic fashion and thus improve our science. As a consequence, reviewers gradually drifted from a rigorous application of the policy to a pro forma determination of the adequacy of including underrepresented racial ethnic minorities, roughly equivalent to whether or not sampling plans assured their representation equal to that characteristic of the settings in which this work was to be conducted. One seldom read arguments, for example, for oversampling special populations in a manner that would truly test the hypotheses underpinning the proposed research, and thereby address race, ethnicity, or disadvantage as possibly important sources of variation in the phenomena under study.

Again, colleagues and I who served on NIH study sections during these years shared this concern and lamented the absence of careful attention to implications for the science. Understanding the original arguments for why, scientifically, such individuals should be included in the work sponsored by $\mathrm{NIH}$ gradually faded; inattention diluted the promise of this remarkable policy. Arguments for the inclusion of underrepresented racial and ethnic minorities and the disadvantaged in $\mathrm{NIH}$-sponsored research eventually shifted from the benefit to science to the underlying importance of health equity and social justice. While the latter are important motives for undertaking such work, they are, in my opinion, necessary, but not sufficient conditions for promoting knowledge acquisition and ensuring the scientific merit of such efforts.

One and one-half decades later, the now National Academy of Medicine reviewed the NIH's strategic research plan to reduce and ultimately eliminate health disparities (Institute of Medicine 2006). This assessment essentially focused on the impact of the National Center on Minority Health and Health Disparities as of 2006, just prior to elevation to its current Institute status. In the Academy's report, the committee, of which I was a member, concluded that the unfinished business of the Center and NIH was to revitalize a focus on the relevant science and to offer a cohering thematic framework for its pursuit. An emphasis on the social determinants of health emerged and gained increasing currency in anticipating and focusing this work, with special attention to culture. As this special issue amply demonstrates, such a framework has played a critical role in organizing attention to the key domains and related constructs that bear importantly on the science underpinning minority health and health disparities research today. 
This special issue, and several recent publications - namely, The Science of Health Disparities Research and Applications (National Institute on Minority Health and Health Disparities 2019) and its companion piece in the American Journal of Public Health (Pérez-Stable and Collins 2019), provide a roadmap by which to guide the relevant science. The articles contained herein illustrate the importance and feasibility of systematic, rigorous inquiry for understanding the specifics of minority health and health disparities. They also convey the importance of the lessons learned for implementation science in general: for discovery, for generalizability, for advancing theory, for enhancing measurement, for improving investigative methods, for promoting attention to neglected areas of research, and for diversifying the scientific work workforce. It returns us to the spirit of 1992, and conviction, albeit now empirically demonstrable, that the work of this nature can be exacting, meritorious, innovative, and broadly relevant. I applaud my colleagues' efforts in this regard and am confident those who follow in their footsteps can now more effectively integrate health equity, social justice, and good science in service of improving the health of racial and ethnic minorities, as well as the disadvantaged, and humankind in general.

In closing, I return to my opening comments about culture, and its essence with respect to understanding as well as enhancing human health and well-being. This final reflection is a cautionary note. A substantial amount of the discussion in this special issue is framed within the context of "decolonizing" our knowledge base, of "framing indigenist research" (Walters et al. 2018). As a Native person and a scientist, I understand and appreciate the underlying issues to which such references give voice. However, these terms are often experienced as confrontational, are frequently polarizing, and therefore not, in my opinion, particularly useful for advancing the scientific agenda. True, presented in this fashion, one may capture an audience's attention, hopefully encourage self-reflection, and even possibly promote critical thinking. All necessary steps to moving the work along these lines toward the goals are stated throughout these articles. Unfortunately, I have also witnessed reactions by many others-fellow scientists, funders, administrators, policy-makers-who miss the message for the medium; colleagues who, blinded by the language, fail to grasp the scientific merits of the positions advocated. Make no doubt, the articles in this special issue are scientifically meritorious. But such phrases foster "pseudo-species like arguments", in which the populations of interest, here American Indians, Alaska Natives, and Native Hawaiians, are deemed so different from other human beings that proponents claim a different science is required to address the questions of the day. Synergies with other investigative methods, other disciplines, and other perspectives can be overlooked, diminishing the opportunity to amplify their impact. The unintended consequence is that colleagues who will most benefit from the lessons to be learned are the least likely to realize them.

As an anthropologist, I find virtually every assertion in this special issue about the epistemological imperatives of our science, about the rich, important variation in the lived experience of Indigenous peoples, and the critical role this experience plays in conceptualizing, designing, and operationalizing effective interventions to be true. Yet these assertions are not limited to American Indians, Alaska Natives, or Native Hawaiians: they speak to all of us, regardless of gender, age, language, religion, or race. Why? Because these assertions are fundamentally about culture and about us as cultural beings. If only the prevention science conducted among non-Indigenist peoples was as thoughtful and penetrating in its implementation as that presented here. Hopefully, someone other than those of us already persuaded as to the intrinsic value of this approach is listening.

\section{Compliance with Ethical Standards}

Conflict of Interest The author declares that he has no conflict of interest.

Ethical Approval This article does not contain any studies with human participants or animals performed by the author.

Informed Consent Because this article is a commentary, informed consent is not applicable.

\section{References}

Dickerson, D., Baldwin, J.A., Belcourt, A., Belone, L., Gittelsohn, J., Kaholokula, J.K., Lowe, J., Patten, C.A., \& Wallerstein, N. (2018). Encompassing cultural contexts within scientific research methodologies in the development of health promotion interventions. Prevention Science. [Epub June 29, 2018.]

Gold, J. (1985). Cartesian dualism and the current crisis in medicine - an appeal for a philosophical approach. Journal of the Royal Society of Medicine, 78, 663-666.

Institute of Medicine. (2006). Committee on the review and assessment of the NIH's strategic research plan and budget to reduce and ultimately eliminate health disparities. In G. E. Thomson, F. Mitchell, \& M. B. Williams (Eds.), Examining the Health Disparities Research Plan of the National Institutes of Health. Unfinished Business. Washington (DC): National Academies Press.

Ivanich, J.D., Mousseau, A.C., Walls, M., Whitbeck, L., Whitesell, N.R. (2018). Pathways of adaptation: Two case studies with one evidence-based substance use prevention program tailored for Indigenous youth. Prevention Science. [Epub June 6, 2018].

Jernigan, V.B.B., D’Amico, E.J., Duran, B., \& Buchwald, D. (2018). Multilevel and community-level interventions with Native Americans: Challenges and opportunities. Prevention Science. [Epub June 6, 2018].

Kirmayer, L. J. (2007). Psychotherapy and the cultural concept of the person. Transcultural Psychiatry, 44(2), 232-257. 
Manson, S. M. (1994). Culture and depression: Discovering variations in the experience of illness. In W. J. Lonner \& R. S. Malpass (Eds.), Psychology and culture (pp. 285-290). Needham: Allyn and Bacon.

Manson, S. M., \& Brenneman, D. L. (1995). Chronic disease among older American Indians: Preventing depression and related problems of coping. In D. K. Padgett (Ed.), Handbook on ethnicity, aging, and mental health (pp. 284-303). Westport: Greenwood Press.

National Institute on Minority Health and Health Disparities, National Institutes of Health. (2019). The Science of Health Disparities Research and Applications. Hoboken: Wiley (In press).

Pérez-Stable, E. J., \& Collins, F. S. (2019). Science visioning in minority health and health dis parities. American Journal of Public Health, 109(S1), S5.

Rasmus, S.M., Whitesell, N.R., Mousseau, A., \& Allen, J. (2018). An intervention science to advance underrepresented perspectives and Indigenous self-determination in health. Prevention Science. [Epub .]
Spoth, R., \& Redmond, C. (2002). Project family prevention trials based in community-university partnerships: Toward scaled-up preventive interventions. Prevention Science, 3, 203-221.221.

Thibaut, F. (2018). The mind-body Cartesian dualism and psychiatry. Dialogues in Clinical Neuroscience, 20(1), 3.

Walters, K.L., Johnson-Jennings, M., Stroud, S., Rasmus, S., Charles, C., John, S., Allen, J., Kaholokula, J.K., Look, M.A., de Silva, M., Lowe, J., Baldwin, J.A., Lawrence, G., Brooks, J., Noonan, C.W., Belcourt, A., Quintana, E., Semmens, E.O., \& Boulafentis, J. (2018). Growing from our roots: Strategies for developing culturally grounded health promotion interventions in American Indian, Alaska Native, and Native Hawaiian communities. Prevention Science. [Epub November 6, 2018].

Publisher's Note Springer Nature remains neutral with regard to jurisdictional claims in published maps and institutional affiliations. 